\title{
Treatment of immune thrombocytopenic purpura: focus on eltrombopag
}

\author{
Lawrence Rice
}

Weill Cornell Medical College, The Methodist Hospital, Houston, Texas, USA
Correspondence: L Rice Professor of Medicine, Weill Cornell Medical College; Chief of Hematology Division, The Methodist Hospital; 6550 Fannin \#I00I, Houston,

TX 77030, USA

Tel $+|7| 3$ 44I 5900

Fax +I 7137937065

Email Irice@tmhs.org

\begin{abstract}
Immune thrombocytopenic purpura (ITP) is a relatively common autoimmune disorder in which antibodies are produced to circulating platelets. Symptoms can be mild, but for most patients the risk of severe bleeding is unacceptable and treatment is required. Glucocorticoids followed by splenectomy had been the mainstays of therapy. High dose intravenous immunoglobulin and anti-RhD therapy are available for patients with severe illness, but produce only temporary benefit. Rituximab may provide more durable responses, danazol may be underutilized, and immunosuppressants and cytotoxic agents are less often required. Recently the pathophysiology of ITP has been more clearly elucidated, particularly the importance of decreased production of platelets in most patients and the very blunted rise that occurs in serum thrombopoietin (TPO). The isolation of TPO and better understanding of its role in thrombopoiesis has led to the development of new highly effective treatments. TPO analogs had some successes in treating highly refractory ITP patients but were taken out of development due to TPO-antibody induction. Two second-generation TPO-mimetics, romiplostim and the orally available eltrombopag, have recently been licensed in some territories for the treatment of ITP. Approval of eltrombopag was based on results from Phase II and III placebo-controlled clinical trials and a long-term extension study. About $80 \%$ of patients achieve significant increases in platelet count ( $11 \%$ of placebo patients), with reduced bleeding and reduced use of concomitant medications; responses are often durable with no tachyphylaxis. The side effects of eltrombopag are generally mild and not worse than placebo, although there are concerns about hepatic dysfunction, and the potentials for thromboses, marrow reticulin fibrosis, rebound thrombocytopenia and cataracts. This is an important new option for highly refractory patients, and its niche in earlier treatment (and for other thrombocytopenic disorders) is yet to be defined.
\end{abstract}

Keywords: eltrombopag, immune thrombocytopenic purpura, autoimmune disorder, serum thrombopoietin

\section{Introduction}

When a teenage girl presented to a Boston hospital in 1945 with bleeding and severe thrombocytopenia, splenectomy was the only known treatment for immune thrombocytopenic purpura (ITP). She was dispatched to the operating room but died from surgical bleeding. This case so profoundly affected a medical student, William Harrington, that he resolved to pursue a career in hematology studying ITP. ${ }^{1}$ There were reasons to suspect that ITP was due to autoantibodies directing the rapid clearance of platelets from the circulation, and Harrington dramatically advanced this theory in 1950 by self-injecting plasma from an ITP patient. His platelet count plummeted, accompanied by petechiae and seizure. Transient thrombocytopenia was then reproduced by giving ITP plasma to various categories of volunteers. ${ }^{2}$ We now know that pathogenic IgG anti-platelet autoantibodies are most often directed against GpIIb-IIIa or GpIb-IX epitopes. ${ }^{3}$

By the mid 1980s, ITP therapy had become straightforward because the pathogenesis was believed to be fully elucidated, the paradigm for humorally-mediated 
autoimmune disease. Glucocorticoid therapies had been developed which paralyzed reticuloendothelial cells that phagocytized antibody-coated platelets, impaired the binding of autoantibody to platelet antigens and, less reliably, diminished the synthesis of anti-platelet antibodies. Steroids were highly effective initially, but the great majority of patients relapsed as the dose was tapered to levels tolerable for the long-term. At that point, splenectomy was performed, impacting the accelerated platelet destruction by removing the major reticuloendothelial organ responsible for platelet clearance, and also a major site for autoantibody production. Short- and long-term disease control were thus achieved in most patients. More potent and toxic immunosuppressive agents, such as cyclophosphamide, vincristine and azathioprine, were used to salvage refractory patients.

In the last two decades, management decisions for ITP have become more complex as new treatment options have emerged. While these options generally achieve disease control, therapy has become more costly and potential toxicities remain problematic. The individual preferences of patients and their physicians have replaced standard treatment algorithms. This is especially true because of a lack of evidence from quality clinical trials, so guidance for clinicians hinges on the vagaries of expert "consensus" opinions. ${ }^{4}$

\section{The disease and current treatments}

ITP is an autoimmune disorder manifest as thrombocytopenia related to the production of antibodies against platelet antigens. It is relatively common in any hematology referral practice, with estimated prevalence 300,000 to 600,000 among adults in the US..$^{5-7}$ Terminology can be confusing and inconsistent: many separate "secondary ITP" from the primary idiopathic form. Secondary ITP may be seen with systemic lupus erythematosus, anti-phospholipid antibodies, HIV, hepatitis C, Helicobacter pylori, lymphoproliferative and other disorders. Several of these are routinely screened for in a patient with isolated thrombocytopenia. The pathophysiology and management decisions with some of these disorders may be the same as with idiopathic ITP. On the other hand, "childhood ITP," often called "acute ITP," behaves differently. Childhood ITP occurs almost as frequently as adult ITP, often follows a viral infection, and usually resolves within 2 months even without therapy. Opinions vary on when and how to treat childhood ITP, with studies challenged by the low incidence of serious morbidity and mortality. ${ }^{8}$ Childhood ITP is not further discussed herein.

A minority of patients with ITP have only mild asymptomatic thrombocytopenia which requires no therapy and may remain stable for years or spontaneously remit. For most patients, platelet levels below $30 \times 10^{9} / \mathrm{L}$ indicate an unacceptable risk of bleeding and so treatment is mandated.

Corticosteroids are the mainstay of initial therapy, with prednisone $1 \mathrm{mg} / \mathrm{kg}$ daily widely employed. Platelets rise to a safe level in more than $80 \%$ to $90 \%$ of patients. However, platelet counts fall back in $90 \%$ of responders as the steroids are tapered. It is an art of the practitioner to transition ITP patients off corticosteroids to other modes of disease control before cumulative steroid toxicities become onerous. Some recent studies have advocated high dose pulses of dexamethasone to achieve durable remissions with lessened toxicity,, 10 but this has not gained widespread popularity. The most recent multi-center trial found long-term disease control in only $39 \%$ (see rituximab below). ${ }^{11}$

Opinions vary widely among experts on when and for how long to employ high-dose intravenous immunoglobulin (IVIG) or anti-RhD. In my practice, IVIG is reserved for unusually severe cases (substantial mucosal and other bleeding; platelets $5 \times 10^{9} / \mathrm{L}$ or less) or special circumstances such as pregnancy or pre-operative. The same applies to anti-D therapy for the $80 \%$ of the population who are $\mathrm{RhD}$ positive and have intact spleens. An advantage of these therapies is rapid action, often increasing platelets within a couple of days in the $80 \%$ who are responsive. The main drawback, besides expense, is that responses are generally very transient, platelets generally returning to baseline levels in 3 weeks. IVIG therapy entails repeated prolonged intravenous infusions, infusional reactions, renal toxicities and, more rarely, aseptic meningitis and thrombotic reactions. Anti-D causes some degree of hemolytic anemia, uncommonly severe. ${ }^{12}$

To maintain remission as steroids are tapered, reasonable options are splenectomy, danazol or rituximab. A decade ago, most adults underwent splenectomy for long-term disease control, often performed sooner rather than later. A recent trend is to reserve surgery for patients who have failed two or more therapies. Splenectomy may also be delayed because of the increasingly recognized possibility of spontaneous remissions, even late, something that also colors the indications and interpretation of results for other therapies. ${ }^{13}$ Nevertheless, results are as good as ever, with $10 \%$ to $40 \%$ never requiring additional therapies..$^{14}$ The availability of laparoscopic surgery reduces immediate morbidity, ${ }^{15}$ but there is lifelong increased risk for serious infection by encapsulated organisms.

Rituximab, an anti-CD20 monoclonal antibody, is increasingly popular as a second-line therapy. Overall responses are obtained in $53 \%$ to $69 \%$ of patients, with complete responses 
in $33 \%$ to $54 \%$, usually beginning about 3 weeks after therapy initiation. ${ }^{16-18}$ The only common side-effects are infusional reactions. The medication is expensive, but responses are often durable, with one study finding that two-thirds of remitting patients are still in remission at 5.8 years, ${ }^{19}$ and another finding 5-year relapse-free and treatment-free survivals of $72 \%$ and $61 \% .{ }^{18}$ In a recently completed randomized trial, a strategy of an initial dexamethasone pulse with rituximab led to sustained responses in $85 \%$ of ITP patients, more than twice the rate with dexamethasone alone. ${ }^{11}$

Danazol, an attenuated androgen, has been successfully used for ITP for more than 25 years, yet is another therapy that engenders particularly diverse expert opinions. ${ }^{4}$ Some believe it underutilized, producing responses in two-thirds of patients in large published case series, including half of patients with highly refractory disease. Responses are often durable, although half require indefinite maintenance; toxicity and costs are modest. ${ }^{20,21}$ Among several unproven theories about its mechanism of action, particularly intriguing is the possibility that it increases thrombopoietin levels, just as androgens were used to increase erythropoietin levels in renal anemia before the advent of the recombinant hormone. ${ }^{22}$ This mechanism of danazol action would predict a high response rate to thrombopoietin (TPO)-mimetic agents, as the more specific purified hormone would be expected to induce higher responses and less toxicity.

Immunosuppressive and cytotoxic therapies are used less commonly, mainly to salvage severely affected and highly refractory patients. ${ }^{23}$ Such therapies have included cyclophosphamide, azathioprine and vincristine, but newer agents such as cyclosporine A and mycophenolate mofetil are now often chosen more readily. Enthusiasm in the $1970 \mathrm{~s}$ and 1980s for vinca-loaded platelets and immunoadsorption with protein A columns ${ }^{24}$ has been discarded. Plasma exchange can be used in desperate situations, but is not usually effective.

\section{Thrombopoietic hormones}

For half a century, studies have suggested the existence of TPO, a hormone that is the main regulator of the circulating platelet mass. When the oncogene c-mpl was discovered, there were reasons to suspect it was the receptor for TPO. Using different strategies, several investigators virtually simultaneously isolated TPO in $1994 .{ }^{25}$ Recombinant TPO quickly entered clinical trials, mainly for chemotherapy-induced thrombocytopenia, as did a pegylated truncated analog, recombinant human megakaryocyte growth and development factor (MGDF). Progress in this field came to an abrupt halt when some volunteers given MGDF developed anti-TPO antibodies that caused long-lasting thrombocytopenia. ${ }^{26,27}$ Subsequently, new TPO-mimetics have been developed that activate the TPO receptor but share no structural homology with native TPO, thus carrying no risk of stimulating the production of anti-TPO neutralizing antibodies.

\section{Rationale for TPO-mimetics in ITP}

Dogma held that the pathophysiology of ITP was simple and straightforward. Autoantibodies bind platelets, directing their accelerated destruction by reticuloendothelial phagocytes. Effective therapies influenced the clearance of coated platelets or reduced autoantibody production.

In clinging to this model, it was necessary to discount or disregard evidence accumulated over decades that platelet production was also impaired with ITP. Countering the notion that a compensatory increase in platelet production should accompany ITP, turnover studies employing radio-labeled autologous platelets revealed substantial production problems in a high percentage of ITP patients. ${ }^{28-31}$ One mechanism for this was the finding that platelet autoantibodies could have effects on megakaryocyte maturation and survival. ${ }^{9,32-34}$ The isolation of TPO allowed accelerated comprehension of the normal regulation of platelet mass. TPO is constitutively produced by the liver and is cleared via binding to receptors on circulating platelets and megakaryocytes. Serum TPO levels become markedly elevated with thrombocytopenia resulting from aplastic anemia or cytotoxic chemotherapy because the reduced platelet and megakaryocyte mass fail to clear the hormone. In contrast, TPO levels are not substantially increased in ITP patients. ${ }^{35,36}$ This provided further rationale for trials of pharmacologic TPO for ITP. Impetus was also provided by initial clinical successes with first-generation TPO-mimetic agents in ITP. I have reported a patient with a treatment-refractory cyclic variant of ITP who responded to MGDF therapy. ${ }^{37}$ This patient has been maintained in remission for 11 years with TPO-mimetic therapy. ${ }^{38}$ Nomura treated four refractory ITP patients with MGDF, three of whom responded. ${ }^{39}$ MGDF also benefited HIV-infected chimpanzees with thrombocytopenia and platelet autoantibodies. ${ }^{40}$

\section{Second-generation TPO-mimetic studies}

The first second-generation TPO-mimetic to enter clinical trials was romiplostim (AMG-531; Nplate).$^{41}$ Romiplostim is an engineered peptibody with four c-mpl binding peptides linked to an immunoglobulin Fc carrier domain. When administered to normal volunteers, romiplostim increases platelet counts 
in a dose-dependent manner at doses above $1 \mu \mathrm{g} / \mathrm{kg}$. As with TPO and other agents that stimulate platelet production through c-mpl binding, the initial rise in platelets occurs in 5 to 7 days, peaks at 2 weeks, then falls to baseline by 3 to 4 weeks. Phase II and Phase III clinical trials in ITP had eligibility and response criteria similar to those of the eltrombopag studies (discussed in the following section) and found that $83 \%$ of patients showed a response, which was sustained and durable in 49\%, regardless of splenectomy status. ${ }^{42,43}$ An extension trial has followed 142 patients for up to 3 years with responses in $90 \%$ of patients, $67 \%$ of platelet counts in the target range, and no evidence of tachyphylaxis. ${ }^{44}$ Not only was bleeding reduced, but quality of life measurements improved in treated patients. ${ }^{45}$

Reported adverse events were generally mild and occurred no more often than with placebo. There are, however, some particular safety concerns that continue to be monitored. One is reticulin fibrosis of the bone marrow; megakaryocyte stimulation predictably causes cytokine release (particularly TGF- $\beta$ ) that can stimulate marrow fibroblasts. ${ }^{46}$ Increased reticulin has been seen in $3.5 \%$ of treated patients, although rarely severe enough to affect blood counts or blood smears. It is reversible on stopping the drug (4 of 271 patients stopped therapy for this reason), and it is not clear how different the incidence is from the ITP patient population in general. Exacerbation of thrombotic tendencies is a valid concern with any treatment for ITP, but incidence of thromboemboli has not been noticeably different from controls. A transient worsening of thrombocytopenia on drug withdrawal has been observed in almost $10 \%$ of patients, but severe bleeding complications accompanying this have not been problematic.

Based on the efficacy and safety data, the FDA approved romiplostim for ITP patients who had failed at least one prior therapy, and restricted its use to prescribers and facilities registered with the NEXUS program. The approved initial dose is $1 \mu \mathrm{g} / \mathrm{kg}$ subcutaneously once weekly, with escalation permitted up to $10 \mu \mathrm{g} / \mathrm{kg}$ based on platelet response.

\section{Eltrombopag}

Eltrombopag (Promacta ${ }^{\circledR}$; GlaxoSmithKline) is an orally available synthetic non-peptide small molecule that binds the transmembrane portion of c-mpl (unlike native TPO or romiplostim), thus activating JAK2 and STAT pathways. Animal studies are hampered by the fact that rodents and dogs do not respond with an increase in platelet count. At high doses ( 2 weeks of $40 \mathrm{mg} / \mathrm{kg} /$ day in rodents and $30 \mathrm{mg} / \mathrm{kg} /$ day in dogs) or with prolonged administration for up to 1 year in dogs, the drug was well-tolerated. Cataracts, renal and hepatic toxicities were seen in animals after 2 years of use. There was no immunogenicity, teratogenicity or carcinogenicity. Three chimpanzees doubled their platelet counts after 5 days of $10 \mathrm{mg} / \mathrm{kg} .{ }^{47}$

Thirteen Phase I studies enrolled 568 patients, including studies of renal or hepatic impaired patients, drug-drug interactions, and cytochrome polymorphisms. ${ }^{48}$ Peak plasma levels are achieved 2 to 6 hours after oral administration and the plasma elimination half-life is 21 to 32 hours. In one placebocontrolled, escalating dose study of 73 men, daily doses of $30 \mathrm{mg}, 50 \mathrm{mg}$ and $75 \mathrm{mg}$ for 10 days raised platelet counts beginning at day 8 , peaking at day 16 , and returning to baseline day 22.; this time course mirrors other TPO-mimetics. Mean initial platelet counts of $239 \times 10^{9} / \mathrm{L}$ rose by $24 \%, 43 \%$ and 50\%, respectively. Lower doses (5, 10 and $20 \mathrm{mg}$ ) showed no difference in platelet counts from placebo. ${ }^{49}$

The Phase II program included trials for ITP, thrombocytopenia with hepatitis $\mathrm{C}(\mathrm{HCV})$, and chemotherapy-induced thrombocytopenia. Thrombocytopenia is common with advanced HCV and contributing factors can include platelet sequestration from portal hypertension/hypersplenism, an immune reaction (ITP-like), and low TPO production from hepatic insufficiency. Effective treatments for $\mathrm{HCV}$ can be myelosuppressive, so exacerbation of the baseline thrombocytopenia frequently prevents optimal therapeutic dosing. Seventy-four patients with $\mathrm{HCV}$-related cirrhosis and platelet counts of $20 \times 10^{9} / \mathrm{L}$ to $70 \times 10^{9} / \mathrm{L}$ were randomized to placebo, $30 \mathrm{mg}, 50 \mathrm{mg}$ or $75 \mathrm{mg}$ of eltrombopag daily. By week four, platelet counts above $100 \times 10 \% / \mathrm{L}$ were achieved in $0 \%, 75 \%$, $79 \%$ and $95 \%$ of patients, respectively, allowing most patients at the two highest doses to tolerate 12 -week courses of peginterferon and ribavirin versus $6 \%$ on placebo. ${ }^{50}$

In a Phase II adult ITP study, 118 patients with platelet counts less than $30 \times 10^{9} / \mathrm{L}$ having failed at least one prior therapy were randomized to placebo, $30 \mathrm{mg}, 50 \mathrm{mg}$ or $75 \mathrm{mg}$ of eltrombopag daily. The lowest eltrombopag dose was ineffective. More than $80 \%$ of patients taking the two higher doses had an increase in platelets by day 15 ; median platelet counts of $128 \times 10^{9} / \mathrm{L}$ and $183 \times 10^{9} / \mathrm{L}$ were seen on day 43 (versus $16 \times 10^{9} / \mathrm{L}$ for placebo patients). Responding patients experienced decreased bleeding events. Serious and overall adverse events were no more common with eltrombopag than with placebo. ${ }^{51}$

Data from the Phase III trial (RAISE) and an extension trial (EXTEND) are recently available from presentations at a national meeting, a publication in Lancet, and material provided to the FDA by the manufacturer. ${ }^{48,52-55}$ Included in RAISE were again ITP patients with platelets less than $30 \times 10^{9} / \mathrm{L}$ who had failed at least one prior therapy; 197 patients were randomized 
in a ratio of 1:2 to placebo or eltrombopag $50 \mathrm{mg}$ daily, with escalation to $75 \mathrm{mg}$ daily allowed. The primary endpoint of platelet count above $50 \times 10^{9} / \mathrm{L}$ was achieved by $59 \%$ on eltrombopag compared to $16 \%$ on placebo $(p<0.01)$. Splenectomy status was not a determinant of response. Bleeding was reduced in treated patients, even in those who did not meet criteria for a platelet response, and several treated patients were able to have surgery without the use of rescue medications. More than 200 patients have been enrolled in the EXTEND trial, with $24 \%$ maintaining a platelet response for more than 6 months.

Safety data were reported to the FDA on more than one thousand exposed individuals of whom more than 300 were ITP patients and more than 200 were treated for at least 6 months (up to 2 years). The most common adverse events were mild (such as headache) and were not more frequent than the incidence with placebo. Phase II studies revealed hepatobiliary abnormalities in $9 \%$ to $10 \%$ of eltrombopag-treated patients compared with $8 \%$ of placebo patients; in the RAISE trial, liver function tests were abnormal in 13\% compared with $7 \%$ of controls. One patient in the trials developed grade 4 hepatic dysfunction, had confounding factors as to the etiology of liver disease, and died of cardiorespiratory failure and pulmonary emboli. A "black box" warning of possible hepatic dysfunction appears on the package label. Cataracts developed or worsened in $5 \%$, compared with $3 \%$ of placebo patients (many patients had significant corticosteroid use). Eleven thromboembolic events have been seen on eltrombopag, one fatal, but all patients had other risk factors, and the rate did not seem to be excessive. Furthermore, platelet function and overall survival appeared normal during therapy. The possibility that platelet counts could fall below baseline with interruption of the drug ("rebound thrombocytopenia") was analyzed. Such transient platelet falls occurred in $30 \%$; in $11 \%$, the platelet count fell to $\leq 10 \times 10^{9} / \mathrm{L}$ and more than $10 \times 10^{9} / \mathrm{L}$ below baseline. While serious bleeding was not seen with these falls, administration of rescue medications in some could have masked or prevented problems. Persistent blood smear abnormalities suggesting significant myelofibrosis were not encountered in any patient. Surveillance bone marrows performed 1 year into treatment revealed mild reticulin fibrosis in 7 of 19 subjects, at least 2 of whom had fibrosis present on pretreatment; this is not clearly increased over what has been reported in other ITP patients and no patient stopped therapy for this reason.

In comparing eltrombopag to romiplostim, they are structurally totally different, they bind c-mpl at different sites causing activation, one is available orally and the other is injected subcutaneously. Response rates and durability seem similar in studies to date. All TPO-mimetics engender at least theoretic
Table I Therapeutic options for immune thrombocytopenic purpura

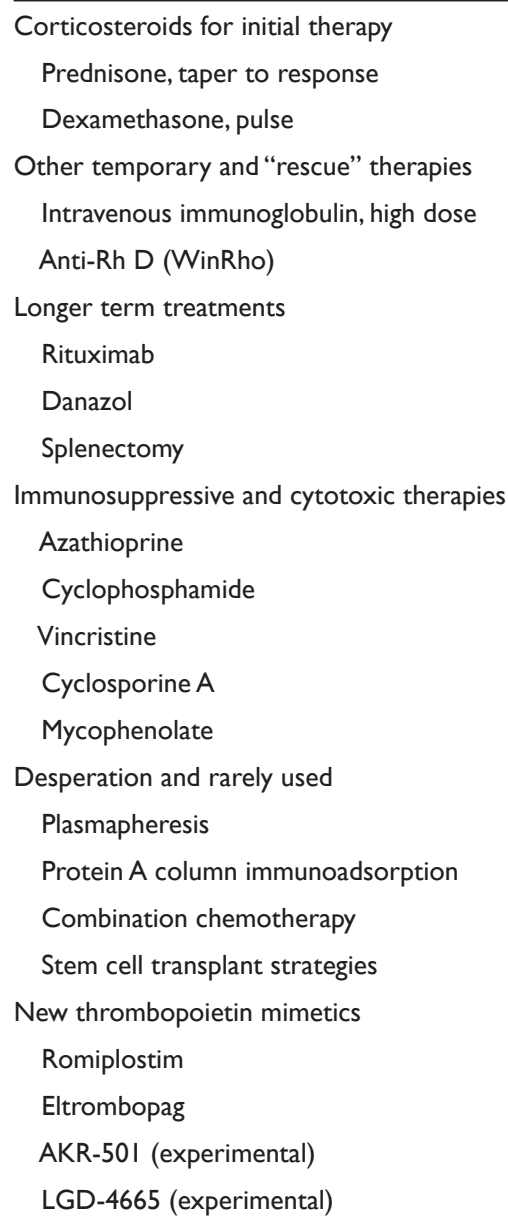

concerns about reticulin fibrosis of the marrow, increasing thrombotic risks, and "rebound" worsening of thrombocytopenia. These potential problems have not emerged as serious problems with either agent. It is not clear whether the relative frequencies of these effects differ between one agent and the other, because of the small number of patients studied, the fact that some of these problems may be related to relative dose, and the differing vigor with which some subclinical changes have been sought. Possible hepatotoxicity has been more appreciated with eltrombopag.

Based on efficacy and safety data, the FDA approved eltrombopag in November 2008 for ITP patients who had failed at least one prior therapy. It is available on a restricted access program (Promacta Cares).

\section{Conclusions and future directions}

ITP is a relatively common blood disorder related to the production of anti-platelet antibodies. Antibody-coated platelets are rapidly cleared from the circulation, so a variety of therapies have been successful that block the increased 
platelet destruction. It is now clear that platelet production is also substantially impaired in most patients. The impaired production results from antibody effects on megakaryocytes and their progenitors, and also to blunted endogenous TPO response resulting from ongoing receptor-mediated clearance of the hormone. After the cloning of TPO and analogs, there were reported therapeutic successes in a few refractory ITP patients, but neutralizing antibodies led to the withdrawal of first-generation TPOs from development. Second-generation TPO-mimetics are now available that stimulate c-mpl but share no homology with the native hormone.

While most patients with ITP do well in the long term on previously available therapies, they may suffer significant toxicities. Second-generation TPO-mimetics have shown responses in $50 \%$ to $80 \%$ of ITP patients with only modest toxicity, and thus they offer another therapeutic option. The first of these agents to enter clinical trials and to be approved by the FDA is romiplostim, a once weekly subcutaneous peptibody. Eltrombopag is the second FDA-approved TPO-mimetic and has the advantage of oral formulation. Newer oral TPO-mimetics in clinical trials include AKR-501 and LGD-4665. Beyond ITP, TPO-mimetics are being studied in chemotherapy-induced thrombocytopenia, myelodysplastic syndromes, hepatitisassociated thrombocytopenia, and other disorders in which it is desirable to stimulate thrombopoiesis. Some concerns persist on the potential of these agents to cause increased thrombosis risk, rebound thrombocytopenia on drug withdrawal, reticulin fibrosis of the marrow, and induction of malignancy, but these have not emerged as major problems in clinical trials. Secondgeneration TPO-mimetics are "miracle drugs" for some highly refractory ITP patients. Given cost considerations and the need for indefinite therapy, it will be interesting to see how large a niche these agents will find in the less refractory ITP patient, and also what their utility will be in other disorders.

\section{Disclosures}

The author receives honoraria for speaking from Amgen (romiplostim) and GSK (argatroban).

\section{References}

1. Altman, LK. Who goes first. The story of self experimentation in medicine. 1st. Ed. New York: Random House; 1987.

2. Arimura G, Harrington WJ, Minnich V. The autoimmune thrombocytopenias. Prog Hematol. 1956;1:166-192.

3. McMillan R. Antiplatelet antibodies in chronic adult immune thrombocytopenic purpura: assays and epitopes. J Pediatr Hematol Oncol. 2003;25 Suppl 1:S57-S61.

4. George JN, Woolf SH, Raskob GE. Idiopathic thrombocytopenic purpura: a practice guideline developed by explicit methods for the American Society of Hematology. Blood. 1996;88(1):3-46.

5. Seagal JB, Powe NR. Prevalence of immune thrombocytopenia: analyses of administrative data. J Thromb Haemost. 2008;4(11):2377-2383.
6. Fuedjo-Tepie MA, Robinson D, Bennett D. Prevalence of diagnosed chronic immune thrombocytopenic purpura in the US: analysis of a large US claim database: a rebuttal. J Thromb Haemost. 2008;6(4):711-712.

7. Satia JA. Epidemiology of immune thrombocytopenic purpura in the United Kingdom, Germany, and the Netherlands. Blood. 2005;106(Supp1):Abstract 5554.

8. Blanchette V, Bolton-Maggs P. Childhood immune thrombocytopenic purpura: diagnosis and management. Pediatr Clin North Am. 2008;55(2):393-420.

9. Chang M, Nakagawa PA, Williams SA, et al. Immune thrombocytopenic purpura (ITP) plasma and purified ITP monoclonal autoantibodies inhibit megakaryocytopoiesis in vitro. Blood. 2003;102(3):887-895.

10. Mazzucconi MG, Fazi P, Bernasconi S, et al; for Gruppo Italiano Malattie Ematologiche dell'Adulto (GIMEMA) Thrombocytopenia Working Party. Therapy with high-dose dexamethasone (HD-DXM) in previously untreated patients affected by idiopathic thrombocytopenic purpura: a GIMEMA experience. Blood. 2007;109(4):1401-1407.

11. Zaja F, Baccarani M, Mazza $P$, et al. A prospective randomized study comparing rituximab and dexamethasone vs dexamethasone alone in ITP: Results of final analysis and long term follow up. Blood. 2008;112(suppl):Abstract 1.

12. AR Gaines. Acute onset hemoglobinemia and/or hemoglobinuria and sequelae following Rho(D) immune globulin intravenous administration in immune thrombocytopenic purpura patients. Blood. 2000;95:2523-2529.

13. Simanek R, Panzer S, Lechner K, Pabinger I. Late spontaneous remissions in severe adult autoimmune thrombocytopenia. Ann Hematol. 2007;86:705-710.

14. Kojouri K, Vesely SK, Terrell DR, George JN. Splenectomy for adult patients with idiopathic thrombocytopenic purpura: a systematic review to assess long-term platelet count responses, prediction of response, and surgical complications. Blood. 2004;104(9):2623-2634.

15. Dolan JP, Sheppard BC, DeLoughery TG. Splenectomy for immune thrombocytopenic purpura: surgery for the 21 st century. Am J Hematol. 2008;83(2):93-96.

16. Arnold DM, Dentali F, Crowther MA, et al. Systematic review: efficacy and safety of rituximab for adults with idiopathic thrombocytopenic purpura. Ann Intern Med. 2007;146(1):25-33.

17. Zhou Z, Yang R. Rituximab treatment for chronic refractory idiopathic thrombocytopenic purpura. Crit Rev Oncol Hematol. 2008;65(1):21-31.

18. Medeot M, Zaja F, Vianelli N, et al. Rituximab therapy in adult patients with relapsed or refractory immune thrombocytopenic purpura: longterm follow-up results. Eur J Haematol. 2008;81(3):165-169.

19. Patel V, Mihatov N, Cooper N, Stasi R, Cunningham-Rundles S, Bussel JB. Long term follow-up of patients with immune thrombocytopenic purpura whose initial response to rituximab lasted a minimum of 1 year. Blood (suppl). 2006;Suppl:abstract 479.

20. Kim SW, Rice L, McCarthy JJ. Efficacy of danazol with autoimmune thrombocytopenia. Clin Appl Thrombosis/Hemostasis. 1997;3:251-255.

21. Maloisel F, Andrès E, Zimmer J, et al. Danazol therapy in patients with chronic idiopathic thrombocytopenic purpura: long-term results. Am J Med. 2004;116(9):590-594.

22. Rice L. Danazol, idiopathic thrombocytopenic purpura, and thrombopoietin. Am J Med. 2004;117(12):972-973.

23. George JN, Kojouri K, Perdue JJ, Vesely SK. Management of patients with chronic, refractory idiopathic thrombocytopenic purpura. Semin Hematol. 2000;37(3):290-298.

24. Snyder HW Jr, Cochran SK, Balint JP Jr, Bertram JH, Mittelman A, Guthrie TH Jr, Jones FR. Experience with protein A-immunoadsorption in treatment-resistant adult immune thrombocytopenic purpura. Blood. 1992;79:2237-2245.

25. Kaushansky K. The molecular mechanisms that control thrombopoiesis. J Clin Invest. 2005;115(12):3339-3347.

26. Kuter DJ, Begley CG. Recombinant human thrombopoietin: basic biology and evaluation of clinical studies. Blood. 2002;100(10): 3457-3469.

27. Li J, Yang C, Xia Y, et al. Thrombocytopenia caused by the development of antibodies to thrombopoietin. Blood. 2001;98(12):3241-3248. 
28. Heyns AD, Lotter MG, Badenhorst PN, et al. Kinetics and sites of destruction of ${ }^{111}$ Indium-oxine-labeled platelets in idiopathic thrombocytopenic purpura: a quantitative study. Am J Hematol. 1982;12(2):167-177.

29. Ballem PJ, Segal GM, Stratton JR, et al. Mechanisms of thrombocytopenia in chronic autoimmune thrombocytopenic purpura. Evidence of both impaired platelet production and increased platelet clearance. J Clin Invest. 1987;80(1):33-40.

30. Siegel RS, Rae JL, Barth S, et al. Platelet survival and turnover: important factors in predicting response to splenectomy in immune thrombocytopenic purpura. Am J Hematol. 1989;30(4):206-212.

31. Louwes H, Zeinali Lathori OA, Vellenga E, de Wolf JT. Platelet kinetic studies in patients with idiopathic thrombocytopenic purpura. Am J Med. 1999;106(4):430-434.

32. Hoffman R, Zaknoen S, Yang HH, et al. An antibody cytotoxic to megakaryocyte progenitor cells in a patient with immune thrombocytopenic purpura. N Engl J Med. 1985;312(18):1170-1174.

33. Takahashi R, Sekine N, Nakatake T. Influence of monoclonal antiplatelet glycoprotein antibodies on in vitro human megakaryocyte colony formation and proplatelet formation. Blood. 1999;93(6):1951-1958.

34. McMillan R, Wang L, Tomer A, Nichol J, Pistillo J. Suppression of in vitro megakaryocyte production by antiplatelet autoantibodies from adult patients with chronic ITP. Blood. 2004;103(4):1364-1369.

35. Emmons RVB, Reid DM, Cohen RL, Meng G, Young NS, Dunbar CE, Shulman RE. Human thrombopoietin levels are high when thrombocytopenia is due to megakaryocyte deficiency and low when due to increased platelet destruction. Blood. 1996;87:4068-4071.

36. Nichol JL. Endogenous (e)TPO levels in health and disease. Possible clues for therapeutic intervention. Stem Cells. 1998;16(suppl 2):165-175.

37. Rice L, Nichol JL, McMillan R, Roskos LK, Bacile M. Cyclic immune thrombocytopenia responsive to thrombopoietic growth factor therapy. Am J Hematol. 2001;68(3):210-214.

38. Bose P, Hussein KK, Terrell DR, Berger D, Rice L, George JN. Successful treatment of cyclic thrombocytopenia with thrombopoietin-mimetic agents: A report of two patients. [submitted].

39. Nomura S, Dan K, Hotta T, et al. Effects of pegylated recombinant human megakaryocyte growth and development factor in patients with idiopathic thrombocytopenic purpura. Blood. 2002;100(2):728-730.

40. Harker LA, Marzec UM, Novembre F, et al. Treatment of thrombocytopenia in chimpanzees infected with human immunodeficiency virus by pegylated recombinant human megakaryocyte growth and development factor. Blood. 1998;91(12):4427-4433.

41. Rice L, Drug evaluation: AMG-531 for the treatment of thrombocytopenias, Curr Opin Invest Drugs. 2006;7(9):834-841.

42. Bussel JB, Kuter DJ, George JN, et al. AMG 531, a thrombopoiesisstimulating protein, for chronic ITP. $N$ Engl J Med. 2006;355(16): $1672-1681$.
43. Kuter DJ, Bussel JB, Lyons RM. Efficacy of romiplostim in patients with chronic immune thrombocytopenic purpura: a double-blind randomised controlled trial. Lancet. 2008;371(9610):395-403.

44. Bussel JB, Kuter DJ, Pullarkat V, Lyons RM, Guo M, Nichol JL. Safety and efficacy of long-term treatment with romiplostim in thrombocytopenic patients with chronic ITP. Blood. 2008. In press.

45. George JN, Mathias SD, Go RS, et al. Improved quality of life for romiplostim-treated patients with chronic immune thrombocytopenic purpura: results from two randomized, placebo-controlled trials. $\mathrm{Br} J$ Haematol. 2009;144:409-415.

46. Kuter DJ, Bain B, Mufti G, Bagg A, Hasserjian RP. Bone marrow fibrosis: pathophysiology and clinical significance of increased bone marrow stromal fibres. Br J Haematol. 2007;139(3):351-362.

47. Erickson-Miller CL, Delorme E, Tian SS, et al. Preclinical Activity of Eltrombopag (SB-497115), an Oral, Non-peptide Thrombopoietin Receptor Agonist. Stem Cells. 2008. In press.

48. FDA website. http://www.fda.gov/ohrms/dockets/ac/08/briefing/20084366b1-02-GSK.pdf. Accessed 11:00CET 02 December 2008. Copy on file.

49. Jenkins JM, Williams D, Deng Y, et al. Phase 1 clinical study of eltrombopag, an oral, nonpeptide thrombopoietin receptor agonist. Blood. 2007;109(11):4739-4741.

50. McHutchison JG, Dusheiko G, Shiffman ML, et al. Eltrombopag for thrombocytopenia in patients with cirrhosis associated with hepatitis C. N Engl J Med. 2007;357(22):2227-2236.

51. Bussel JB, Cheng G, Saleh MN, et al. Eltrombopag for the treatment of chronic idiopathic thrombocytopenic purpura. $N$ Engl J Med. 2007;357(22):2237-2247.

52. Bussel JB, Provan D, Shamsi T, et al. Effect of eltrombopag on platelet counts and bleeding during treatment of chronic idiopathic thrombocytopenic purpura: a randomised, double-blind, placebo-controlled trial. Lancet. 2009;373:641-648.

53. Bussel JB, Cheng G, Saleh MN, et al. Safety and efficacy of long-term treatment with oral eltrombopag for chronic idiopathic thrombocytopenic purpura. Blood. 2008;112 suppl:Abstract 3432.

54. Cheng G, Bussel, JB, Saleh MN, et al. Eltrombopag delivers clinical benefit in chronic idiopathic thrombocytopenic purpura (ITP) patients not achieving platelet counts $\geq 50,000 / \mu \mathrm{L}$ - data from the EXTEND study. Blood. 2008;112 suppl:Abstract 3430.

55. Saleh MN, Bussel JB, Cheng G, et al. Eltrombopag is efficacious in patients with refractory chronic idiopathic thrombocytopenic purpura (ITP) - data from the EXTEND Study. Blood. 2008;112 suppl:Abstract 401.

56. Promacta: Highlights of Prescribing Information. Available at: http:// www.fda.gov/cder/foi/label/2008/0222911bl.pdf1 
